\title{
Sequence diversification in recessive alleles of two host factor genes suggests adaptive selection for bymovirus resistance in cultivated barley from East Asia
}

\author{
Ping Yang ${ }^{1}$ Antje Habekuß ${ }^{2} \cdot$ Bernhard J. Hofinger $^{3} \cdot$ Kostya Kanyuka $^{3}$. \\ Benjamin Kilian $^{1,4} \cdot$ Andreas Graner $^{1} \cdot$ Frank Ordon $^{2} \cdot$ Nils Stein $^{1}(\mathbb{D})$
}

Received: 16 February 2016 / Accepted: 14 October 2016 / Published online: 9 November 2016

(C) The Author(s) 2016. This article is published with open access at Springerlink.com

\begin{abstract}
Key message Two distinct patterns of sequence diversity for the recessive alleles of two host factors $\mathrm{HvP}$ DIL5-1 and HVEIF4E indicated the adaptive selection for bymovirus resistance in cultivated barley from East Asia.

Abstract Plant pathogens are constantly challenging plant fitness and driving resistance gene evolution in host species. Little is known about the evolution of sequence diversity in host recessive resistance genes that interact with plant viruses. Here, by combining previously published and newly generated targeted re-sequencing information, we systematically analyzed natural variation in a broad collection of wild (Hordeum spontaneum; $\mathrm{Hs}$ ) and domesticated barleys (Hordeum
\end{abstract}

Communicated by X. Qi.

Data deposition: The sequences reported in this paper are deposited in the NCBI database (KT819151-KT819158, KT881104-KT881168). They were released on April 1st, 2016.

Electronic supplementary material The online version of this article (doi:10.1007/s00122-016-2814-z) contains supplementary material, which is available to authorized users.

Nils Stein

Stein@ipk-gatersleben.de

1 Leibniz Institute of Plant Genetics and Crop Plant Research (IPK), OT Gatersleben, 06466 Stadt Seeland, Germany

2 Institute for Resistance Research and Stress Tolerance, Julius Kuehn Institute (JKI), Federal Research Centre for Cultivated Plants, 06484 Quedlinburg, Germany

3 Plant Biology and Crop Science Department, Rothamsted Research, Harpenden AL5 2JQ, UK

4 Present Address: Global Crop Diversity Trust, Platz der Vereinten Nationen 7, 53113 Bonn, Germany vulgare; $H v$ ) using the full-length coding sequence of the two host factor genes, HvPDIL5- 1 and HvEIF4E, conferring recessive resistance to the agriculturally important Barley yellow mosaic virus (BaYMV) and Barley mild mosaic virus (BaMMV). Interestingly, two types of gene evolution conferred by sequence variation in domesticated barley, but not in wild barley were observed. Whereas resistance-conferring alleles of HvEIF4E exclusively contained non-synonymous amino acid substitutions (including in-frame sequence deletions and insertions), loss-of-function alleles were predominantly responsible for the HvPDIL5-1 conferred bymovirus resistance. A strong correlation between the geographic origin and the frequency of barley accessions carrying resistanceconferring alleles was evident for each of the two host factor genes, indicating adaptive selection for bymovirus resistance in cultivated barley from East Asia.

\section{Introduction}

Plant pathogens such as viruses, bacteria and fungi are constantly challenging crop plant fitness since infections may cause yield losses (Oerke 2006). Resistant genotypes have the selective advantage of producing (more) seeds, thus their offspring may predominate in populations after some generations under strong selection. Some pathogen isolates may counteract this via own rapid evolution through mutation - this triggers the 'arms race' or the antagonistic co-evolution between plants and their adapted pathogens (Jones and Dangl 2006; Obbard and Dudas 2014; Rausher 2001). In natural populations or wild species a higher level of genetic diversity could maintain natural resistances that prevent the epidemic outbreak of diseases and their severe consequences for the populations or species (Tanksley and McCouch 1997). By contrast, the monoculture 
in agricultural systems destabilizes the balance, and the densely grown and genetically uniform crop populations facilitate the success of individual newly evolved and virulent strains of a pathogen supporting the chance of pathogen transmission and epidemic outbreak of respective diseases (Stukenbrock and McDonald 2008). Therefore, understanding the patterns of sequence variation underlying the evolution of dominant resistance genes and recessive host factors in cultivated crops and their wild progenitors should contribute to reveal the evolutionary processes during domestication, improvement and local adaptation of crops and thus may provide insight into a more sustainable use of resistance resources in agriculture.

Unlike bacterial and fungal pathogens that maintain relatively large genomes, plant RNA viruses have small genomes and commonly encode only for about four to ten viral proteins. Such viruses cannot complete their entire proliferation cycle independently of their plant hosts. For instance, they are dependent on the host's cellular protein synthesis machinery and also often employ other hostencoded proteins to complete their lifecycle (Ahlquist et al. 2003; Robaglia and Caranta 2006). These host components are known as 'susceptibility' or 'host' factors. Mutations in the genes coding for host factors may result in virus resistance (Soosaar et al. 2005; Whitham and Wang 2004). These recessive resistance genes, therefore, function very differently compared to the dominant resistance genes such as the nucleotide binding leucine rich repeat (NB-LRR) protein encoded genes (Sacristan and Garcia-Arenal 2008), which recognize directly or indirectly the so-called effector proteins produced by the pathogens and then trigger an active defense response often culminating in plant cell death and halting the pathogen growth (Tiffin and Moeller 2006). Depending on the selection pressure imposed by the frequency and/or severity of the respective virus disease, sequence variation in the host genes conferring disease resistance may be quickly selected and maintained at higher frequency in populations under conditions of natural selection. Artificial selection during domestication, adaptive improvement and breeding could even accelerate this selection process. Rapid evolution of plant virus genomes, which occurs constantly at least in RNA viruses due to the lack of an efficient RNA sequence repair system (Domingo and Holland 1997), may result in emergence of new virus pathotypes being able to overcome the newly evolved plant defense mechanism, e.g. by cooperating with alternate host factors or by adapting to the modified host proteins (Kühne et al. 2003; Okada et al. 2003). In agricultural systems, infectious diseases reduce yields and can threaten the sustainability of crop production (Oerke 2006). Selection for resistance forms of host factors was probably unintentional during the early domestication and an adaptation to local environments (Kang et al. 2005). Understanding the natural variation of such host factors could reveal the mechanisms underlying the 'arms race' between cultivated plants and their viruses.

The barley yellow mosaic virus disease, wherever it occurs, is a severe, long-term and persistent biotic stress, threatening winter barley production in a majority of European and Eastern Asian countries (Kühne 2009). It was first detected in Japan in the 1940s (Ikata and Kawai 1940), and later in the 1950s observed in China and Korea (Lee et al. 1996; Zhou and Cao 1985), and since 1978 reported to occur in European countries (Kühne 2009). The disease is caused by infection with isolates of Barley yellow mosaic virus and/or Barley mild mosaic virus, two members of the genus Bymovirus in the Potyviridae family. Under natural conditions, the bymovirus disease is transmitted through barley roots by the soil-borne Plasmodiophorid Polymyxa graminis (Adams et al. 1988). Persistent resting spores of this microorganism provide protection for virus particles, which can remain infectious for many years (Huth 1991). There are currently no chemicals available for control of plant viruses, whereas use of chemical agents (i.e. soil fumigants) effective against the virus vector, $P$. graminis, is undesirable due to ecological implications (Kanyuka et al. 2003; Kühne 2009). After infection, yield losses of up to $50 \%$ have been observed in Europe (Plumb et al. 1986). In Asia, the disease can be even more severe where complete yield losses were recorded in China in the mid-1970s (Chen 2005; Kühne 2009). Breeding for genetic resistance to bymoviruses, therefore, remains the most viable and environmentally safe option for crop protection, and over time eighteen bymovirus resistance loci were genetically characterized in barley (Kai et al. 2012; Ordon et al. 2005). We previously isolated by positional cloning two recessive resistance genes ryml/11 and rym $4 / 5$, corresponding to two different host factor genes Protein Disulfide Isomerase Like 5-1 (HvPDIL5-1) and Eukaryotic Translation Initiation Factor 4E (HvEIF4E), respectively (Kanyuka et al. 2005; Stein et al. 2005; Yang et al. 2014a, b). The resistance genes/alleles rym 1 and rym 5 were originally found in a Chinese landrace "Mokusseko 3" and shown to be inherited independently (Konishi et al. 1997). The HvEIF4E protein putatively functions in assisting the translation initiation of bymovirus precursor protein (Kanyuka et al. 2005; Stein et al. 2005), and HvPDIL5-1 is speculated to function as chaperone in correct folding of virus proteins (Yang et al. 2014b). Our previous work revealed sequence diversity of $H v E I F 4 E$ in domesticated barley accessions and $H v P$ DIL5-1 in wild/domesticated accessions (Hofinger et al. 2011; Yang et al. 2014b). However, the sequence diversity of $H v E I F 4 E$ in wild barley was unknown, and importantly, the responses of most of these HvEIF4E and HvPDIL5-1 haplotypes to the bymovirus disease of barley were not yet investigated. This limited our understanding of the relevance of naturally occurring sequence variation of both host 
factor genes in eco-system vs agricultural system, especially in the regions severely threatened by the bymovirus disease. By combining previously published and newly generated sequences and by testing with a viral assay the identified haplotypes, here we systematically investigated the diversity and frequency of sequence variation patterns in resistanceconferring haplotypes of these two host factor genes in barley genotypes from different geographic regions.

\section{Materials and methods}

\section{Plant materials}

365 wild and 2557 domesticated barleys (mostly landraces or historic cultivars, but also including six-rowed barley with brittle rachis Hordeum vulgare subsp. agriocrithon) with the GPS-coordinated geographical information, which were mainly collected by IPK-Genebank or obtained from the National Small Grains Collection, USDA-ARS (Aberdeen, Idaho, USA), were used in the present study (Supplementary Fig. S1; Supplementary Tables S1, S2). The wild barley accessions were mainly collected from the Near East, and the cultivated barley accessions originated from 89 countries representing most of the barley cultivation areas worldwide. For a small number of accessions the precise collection site was unclear, and for these we specified the latitude and longitude of the capital of the source state/province. Details of the barley accessions and the collection sites (Country, latitude and longitude) are given in Supplementary Table S2.

\section{Amplicon sequencing}

Plant DNA and RNA extraction, synthesis of first-strand cDNA, PCR amplification, purification of PCR products and Sanger sequencing were performed as described previously (Yang et al. 2014b). Purification of PCR amplicons was carried out using the NucleoFast 96 PCR Kit (Macherey-Nagel, Germany). Two $\mu$ l of each purified PCR product was analyzed and quantified by agarose gel electrophoresis. The optimal amount of each purified amplicon (10 ng per each $100 \mathrm{bp}$ ) was subjected to cycle-sequencing and the products analyzed on an ABI-3730xl DNA Analyzer (Applied Biosystems, Darmstadt, Germany). The trimmed sequence reads were assembled using Sequencher 4.7 (Gene Codes, USA). The primers used in this study are given in Supplementary Table S3.

\section{Resistance tests}

Plant material required for performing virus resistance tests was cultivated in climatic chamber as previously described (Habekuß et al. 2008; Yang et al. 2014b). A subset of wild and domesticated barley accessions carrying non-synonymous mutations in either HvPDIL5-1 or HvEIF4E was analyzed after mechanical inoculation with the Aschersleben isolate of BaMMV (BaMMV-ASL). At least 10 plants per accession were tested. At three-leaf stage (2 week after sowing) plants were mechanically inoculated twice at an interval of 5-7 days. Four to six weeks later, the phenotypes were collected by detecting viral RNAs by RT-PCR (via amplification of a 525-bp viral VPg gene fragment), or DAS-ELISA with a viral coat protein-specific antibody (Clark and Adams 1977). For instance, if absorbance at $405 \mathrm{~nm}$ was below 0.1 , the respective plant was scored as being resistant (no virus particles detected). Otherwise, plants were scored susceptible (absorbance $>0.1$ ).

\section{Data analysis}

Sequence assembly and alignments were performed using Sequencher v4.7 (Gene Codes, USA). Sequence reads that originated from introns, and those of poor quality or indication of heterozygosity were removed manually from the analysis. The genotypes for which only the partial gene coding sequences were obtained were also excluded from the analysis. The polymorphic loci including loss-of-function $(L o F)$, synonymous $(S)$ and non-synonymous $(N s)$ were counted manually. The allelic haplotypes, the haplotype diversity $(H)$, the nucleotide diversity $(\pi)$ as well as statistics for selection (Fu and $\mathrm{Li}$ 's $D^{*}$ and $F^{*}$, Tajima's D) were calculated using DNASP v5.10.01 (Librado and Rozas 2009). The generated files, containing polymorphism and haplotype information, were subjected to the Median-Joining network analysis using DNA Alignment v1.3.1.1, Network v4.6.1.1 and Network Publisher v1.3.0.0 software (Fluxus Technology, UK). The significance of type of mutations between wild and domesticated barleys was analysed using Fisher's Exact Test.

The topographic maps were drawn using ArcGIS 10 software (ESRI, Redlands, CA, USA). The Base Map at $10-\mathrm{km}$ resolution developed by the US Geological Survey (USGS) according to the GTOPO30 global digital elevation dataset (http://eros.usgs.gov) was used. These accessions used in this study were assigned to the Base Map according to the specified collection sites (Supplementary Table S2).

\section{Results}

\section{HvPDIL5-1 and HvEIF4E exhibit distinct patterns of sequence diversity in domesticated but not in wild barley}

To assess the extent and pattern of global genetic diversity of the two host factor genes HvPDIL5-1 and HvEIF4E, 
sequence variation in the full-length open reading frames (fl-ORFs) of both genes was surveyed in 365 wild and 2557 domesticated geographically referenced barley accessions (Supplementary Tables S1, S2). The dataset included (i) previously published sequences of $H v E I F 4 E$ obtained from 1090 cultivated barleys (Hofinger et al. 2011) and HvPDIL5-l obtained from 350 and 1382 wild and domesticated barleys, respectively (Yang et al. 2014b), as well as (ii) newly generated sequences of HvPDIL5-1 and HvEIF4E obtained in this study from 856 cultivated and 320 wild barley accessions, respectively (Table 1). Furthermore, we sequenced in a subset of the total of 2922 barley accessions the fl-ORFs of HvGT43 and HvMCT1 (Supplementary Table S1). These genes co-segregated and are located in close physical proximity to HvPDIL5-1 and HvEIF4E, respectively (Fig. 1a, Stein et al. 2005; Yang et al. 2014a, b), and were included in this study for the purpose of determining the demography of population and testing the effect of genomic location on patterns of sequence diversity. The relative genomic positions of HvGT43/HvPDIL5-1 and HvMCT-1/HvEIF4E, besides allocating to different chromosomes, are significantly different. The first gene pair is located close to the centromere (low recombination frequency region) on the long arm of chromosome $4 \mathrm{H}$ (4HL) whereas the second gene pair is located close to the telomere (high recombination frequency region) on the long arm of chromosome 3H (3HL) (IBRC 2012). Importantly, fl-ORF sequences of HvPDIL5-1 and HvEIF4E genes were obtained from a shared set of 1152 genotypes, and fl-ORF sequences of all four genes HvPDIL5-1, HvEIF4E, HvGT43 and HvMCT-1 were obtained from a shared set of 392 barley accessions (Supplementary Table S2).

After removal of low quality data a total of 2588 , 1410,413 and $453 \mathrm{fl}-\mathrm{ORF}$ sequences of the four genes HvPDIL5-1, HvEIF4E, HvGT43 and HvMCT-1 were collected and analyzed for sequence diversity, respectively (Fig. 1; Table 1). A total of 30, 65, 17 and 8 haplotypes, respectively, were defined for HvPDIL5-1, HvEIF4E, HvGT43 and HvMCT-1 (Fig. 2; Supplementary Tables S4-S8). This included two newly identified HvPDIL5-1 haplotypes (hap-XXIX and XXX) and 18 newly identified HvEIF4E haplotypes (hap-XLIV-LXI) (Supplementary Tables S4, S5). One predominating haplotype each was found for HvPDIL5-1 (hap-I, 94.5\%) and for HvMCT-1 (hap-I, 92.3\%), respectively, whereas two and three frequent haplotypes were detected for $H v E I F 4 E$ (hap-wt0A, 57.1\%; hap-I, 17.6\%) and HvGT43 (hap-I, $55.9 \%$; hap-II, $11.6 \%$; hap-III, $25.9 \%$ ) (Supplementary Tables S4-S8).

We further analyzed the coding sequence diversity of these four genes in wild barley in comparison to domesticated barley.

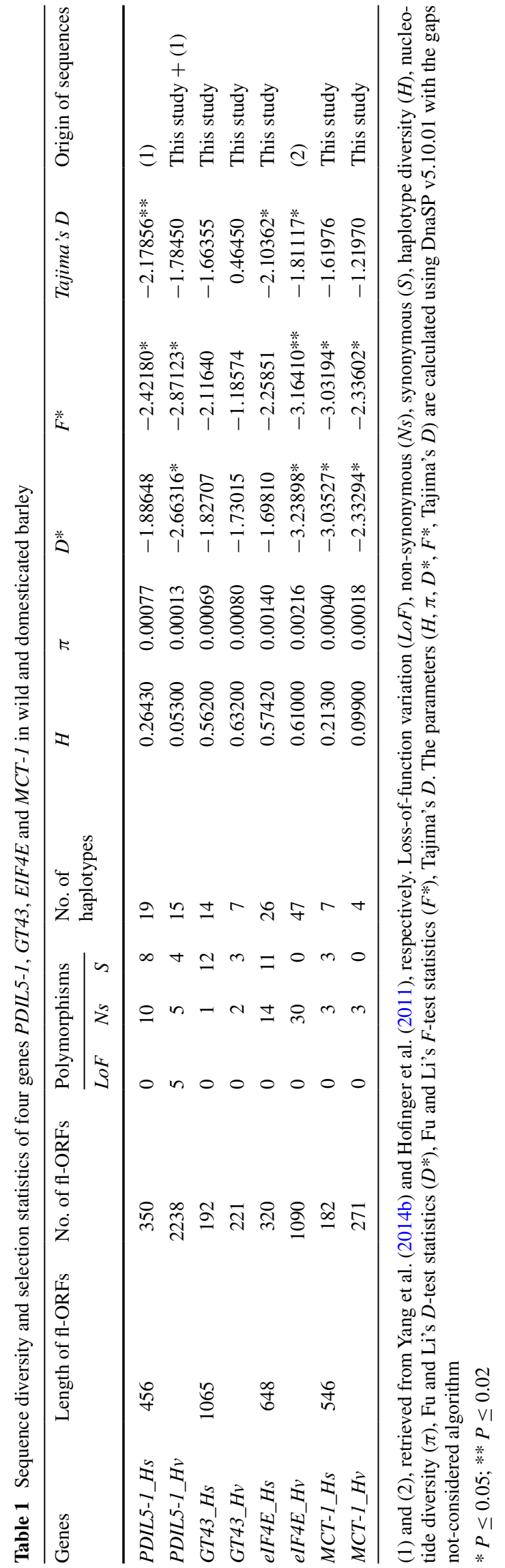



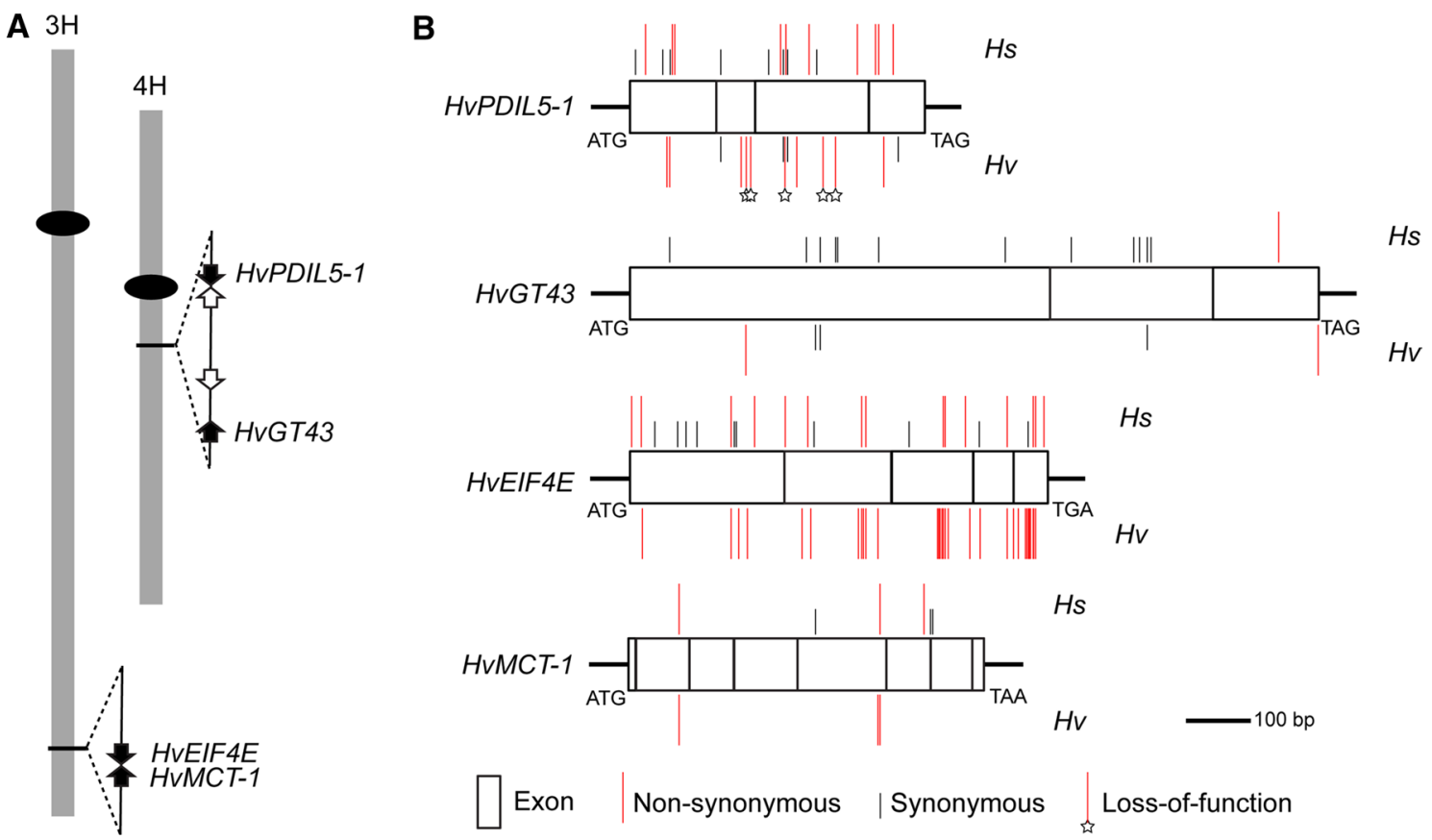

Fig. 1 Sequence variation in HvPDIL5-1,HvGT43, HvEIF4E and HvMCT-1 in wild (Hordeum spontaneum; Hs) and domesticated (Hordeum vulgare; $\mathrm{Hv}$ ) barley. a Physical positions of these four genes on the barley chromosomes $3 \mathrm{H}$ and $4 \mathrm{H}$. The solid black ovals represent centromeres. The distance between $H v E I F 4 E$ and $H v M C T$ 1 is 1048-bp, while the space between HvPDIL5-1 and HvGT43 is about $620 \mathrm{~kb}$. However, no recombination events were detected in

\section{HvPDIL5-1}

Nineteen wild barley haplotypes (350 analyzed sequences) were observed based on ten non-synonymous (refers to non-synonymous amino acid change or in-frame deletions/ insertions) and eight synonymous mutations, while only 15 haplotypes in domesticated barley (from 2238 sequences) were revealed with five containing loss-of-function mutations/deletions, another five containing non-synonymous mutations and remaining four containing synonymous mutations (Table 1). Synonymous and non-synonymous mutations in wild barley occurred evenly along the coding gene sequence, whereas loss-of-function sequence variation in domesticated barley were enriched in the central part of the gene (Fig. 1b), coding putatively for the functional thioredoxin domain (Yang et al. 2014b). Four haplotypes (hap-I, III, IV and XXIV) were shared between wild and domesticated barleys (Fig. 2). Haplotype I predominated in the collection and other haplotypes were derived from this ancestral haplotype by one or two mutations (Yang et al. 2014b). Interestingly, loss-of-function haplotypes were only found to be significantly accumulated in domesticated, but not in wild barley (Fisher's Exact Test, $P=0.0099$ ). The number of polymorphisms, haplotypes, as well as the values of the parameters $H$ and $\pi$ were lower pairs of HvPDIL5-1/HvGT43 and HvEIF4E/HvMCT-1 in thousands of segregating $F_{2}$ lines (Stein et al. 2005; Yang et al. 2014a, b). b Distribution of mutations in the full-length open reading frame (flORFs). The diagrams were drawn according to the length of each flORF. The rectangles within each fl-ORF show positions of gene coding exons

in domesticated barley compared to those in wild barley (Table 1), indicating the reduction of gene sequence diversity. Statistical tests employing $\mathrm{Fu}$ and $\mathrm{Li}$ 's $D^{*}$ and $F^{*}$ and also Tajima`s $D$ indicated low frequencies of rare alleles of HvPDIL5-1 in wild and domesticated barley, possibly being related to selection or population size expansion.

\section{HvGT43}

The close neighbor gene of HvPDIL5- 1 exhibited thirteen and five polymorphic nucleotide positions (including nonsynonymous and synonymous mutations) in 192 wild and 221 domesticated barley accessions, respectively, defining 14 and seven haplotypes (Table 1). Since the same three major haplotypes (hap-I, II and III) were also present in domesticated barley accessions $(95.5 \%, 211$ out of 221 accessions), no strong differences were found for $H$ and $\pi$ values between the domesticated and wild barleys (Table 1). The same set of cultivated barley $v s$ wild barley revealed a strongly decreased sequence diversity at the HvPDIL5-1 locus (Supplementary Table S9). This indicated that the observed sequence diversity of HvPDIL5-1 and HvGT43 was gene specific and irrespective of chromosomal position. Importantly, no defective haplotype was found at the HvGT43 locus. 


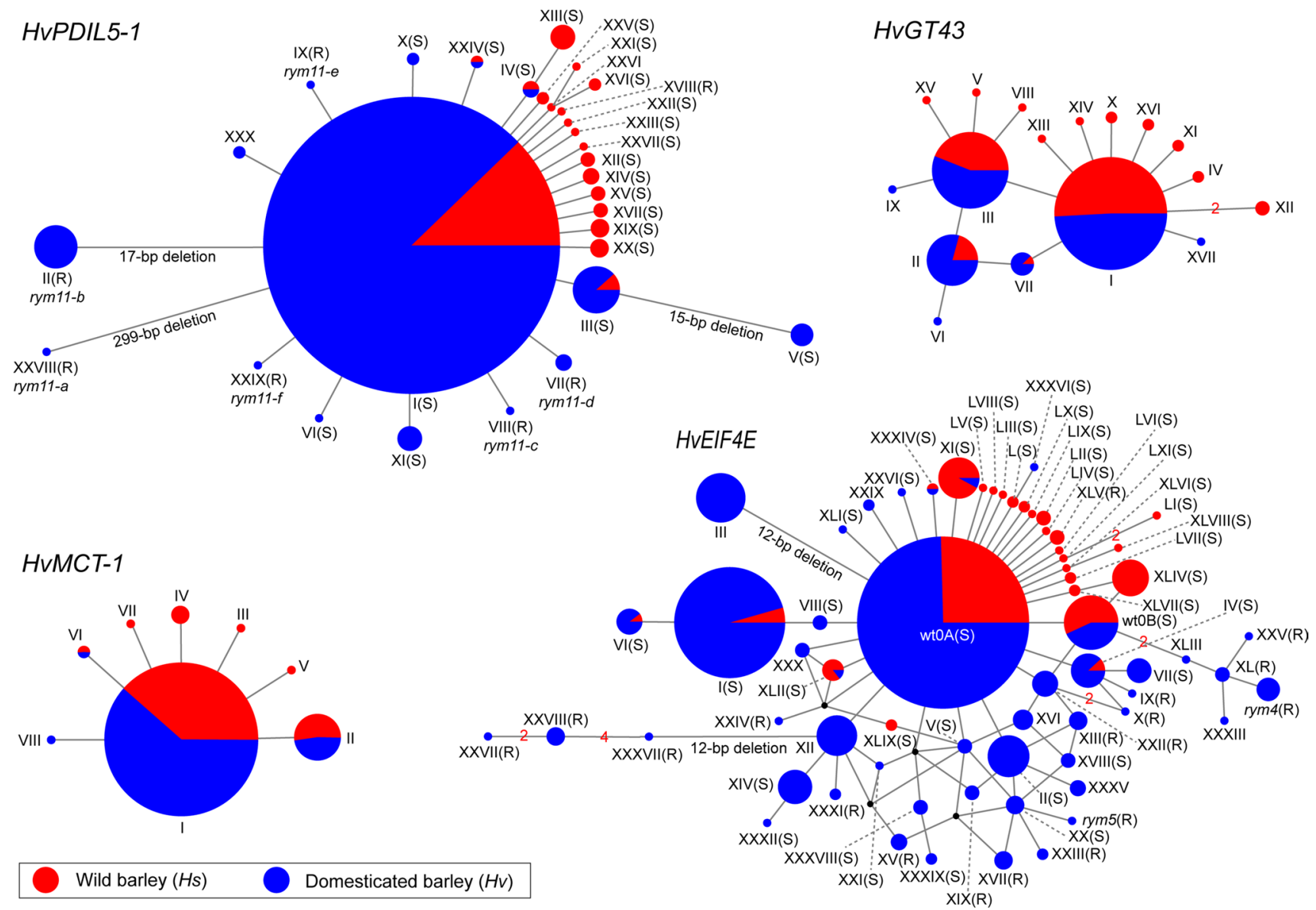

Fig. 2 Haplotype networks of HvPDIL5-1, HvGT43, HvEIF4E and HvMCT-1. Median-Joining (MJ) network was constructed from the haplotypes that were defined by the full-length ORFs. Haplotypes are represented by blue, red and blue/red circles. Size of each circle corresponds to the number of the accessions identified to carry that particular haplotype. Short solid lines represent genetic distances

\section{HvEIF4E}

In 320 wild and 1090 domesticated barley accessions, 26 and 47 haplotypes were defined, respectively. Fourteen nonsynonymous and eleven synonymous nucleotide polymorphisms were detected in wild barley (Table 1), and these were evenly distributed along the coding gene sequence (Fig. 1b). This pattern was similar to the observations at the HvPDIL5-1 locus, which implied a lack of specific selection at a particular region of both genes in wild barley. As to domesticated barley, an extreme situation was observed: sequence diversity was caused by a total of 30 non-synonymous mutations, whereas not even a single silent (synonymous) mutation was detected (Fisher's Exact Test, $P=0.0001$, Table 1). Non-synonymous mutations were enriched at three regions (nucleotides 336-384, 480-528, and 600-648) (Fig. 1b), coding for amino acid sequences located in proximity to the putative cap-binding domain between haplotypes, typically indicating in a single base pair difference. More substantial differences are represented by longer solid lines with the nature of polymorphism indicated below each line. $(R)$ and $(S)$ indicate the bymovirus resistance and susceptibility conferring haplotypes, respectively. For further details of each haplotype please see Supplementary Tables S4-S8 (color figure online)

of the protein (Kanyuka et al. 2005; Stein et al. 2005). In contrast to HvPDIL5-1 that showed a simple evolutionary network, the Median-Joining (MJ) network revealed a complex status of sequence variation for HvEIF4E (Fig. 2). For instance, it seems likely that haplotype XII originated by one additional mutation from one of haplotypes wt0A, XIV, XXI, XXXI or XXXII. The number of polymorphic loci and haplotypes, and $H$ and $\pi$ values in cultivated barley were not lower than in wild barley.

\section{HvMCT-1}

Three synonymous and three non-synonymous mutations of this gene were detected in wild barley accessions, while only three non-synonymous mutations were found in domesticated barley (Table 1). In contrast to HvEIF4E that represented a higher sequence diversity in domesticated barley, the same set of barley accessions revealed 
dramatically decreased sequence diversity at the MCT-1 locus in domesticated $v s$ wild barley (Supplementary Table S9). This indicated that the higher sequence diversity of $H v E I F 4 E$ was not a function of recombination frequency at the $3 \mathrm{HL}$ telomeric region.

Collectively, the sequence variation analyses revealed two qualitatively distinct patterns of gene sequence diversity with a bias for loss-of-function mutations in $H v P$ DIL5-1 and non-synonymous mutations in HVEIF4E-both occurring only in domesticated but not in wild barley accessions. Importantly, analyzing the sequence diversity of the next neighboring genes $H v G T 43$ and HvMCT-1 indicated that the two distinct patterns observed for HvPDIL5-1 and $H v E I F 4 E$ are characteristic for the respective positions on the chromosome.

Bymovirus resistance correlated with the increased diversity of the host factor genes HvEIF4E and HvPDIL5-1.

The barley yellow mosaic virus disease is a highly virulent and persistent biotic stress for cultivated barley in East Asia and Europe (Kühne 2009), and selection for resistance was an important target in recent barley breeding and cultivation in these regions (Chen 2005; Ordon et al. 2005). To determine whether appearance of haplotypes conferring virus resistance was associated with the occurrence of loss-of-function and non-synonymous exchange mutations in HvPDIL5-1 and HvEIF4E (the corresponding recessive alleles of these genes conferring resistance to bymoviruses are known as ryml/11 and rym $4 / 5$, respectively), we tested accessions carrying different haplotypes at the HvPDIL5-1 or HvEIF4E locus for their reaction to a common isolate of Barley mild mosaic virus (BaMMV-ASL). A total of 30 and 65 different haplotypes of HvPDIL5-1 and HvEIF4E, respectively, were defined in wild and domesticated barley accessions (Supplementary Tables S4, S5). Of these, a subset of accessions representative for haplotypes carrying either lossof-function and/or non-synonymous mutations were subjected to mechanical virus inoculation (Supplementary Tables S10, S11). In these assays, primarily the accessions carrying known, previously characterized susceptibility haplotypes at one locus and novel haplotypes with the unknown susceptibility/resistance status at another locus were used. For instance, when one accession contained a known susceptibility haplotype of HvPDIL5-1, testing the susceptibility/resistance status of a novel haplotype of $H v E I F 4 E$ was allowed. However, most of accessions carrying haplotypes of unknown susceptibility/ resistance status at both loci (HvPDIL5-1 and HvEIF4E) were excluded (or the phenotype of respective haplotype was marked as 'unknown'). It needs to be noted, that this approach cannot rule out completely the remote possibility that an accession may contain additional independent bymovirus resistance loci. Less than $1 \%$ of nearly 10,000 domesticated barley accessions were reported to be completely resistant against isolates of BaYMV and BaMMV in the field in earlier studies (Ruan et al. 1984; Zhou and Cao 1985).

\section{HvPDIL5-1}

Seven HvPDIL5-1 haplotypes (hap-II, VII, VIII, IX, XVIII, XXVIII, and XXIX) were classified as resistant to BaMMV-ASL (Fig. 3; Supplementary Table S4). This included a previously undescribed loss-of-function haplotype (G256A/hap-XXIX/ryml1-f) encoding a truncated PDIL5-1-like protein, and a known haplotype (hapXVIII) containing a non-synonymous mutation (A239G/ Glu80Gly) in HvPDIL5-1. Only one wild barley accession carrying hap-XVIII was identified, and it showed resistance to BaMMV-ASL (Supplementary Table S10). This reveals $H$. spontaneum as a useful source of resistance to bymoviruses. In domesticated barley, accessions carrying one of the six HvPDIL5-1 haplotypes, hap-II, VII, VIII, IX, XXVIII, and XXIX, also displayed resistance to BaMMVASL (Fig. 3). These resistance-conferring haplotypes were present in $38(1.69 \%)$ of 2238 cultivated barley accessions (Figs. 3, 4a), and of these 37 were carrying haplotypes containing loss-of-function mutations in HvPDIL5-1 (Supplementary Table S4). Thus, in the case of HvPDIL5-1, it appears that the mutations resulting in a loss of function of the encoded host factor protein are largely responsible for the observed bymovirus resistance phenotype.

\section{HvEIF4E}

Accessions representing either one of the identified nineteen $H v E I F 4 E$ haplotypes were tested as conferring resistance to BaMMV-ASL (Supplementary Tables S5, S11). This included three previously reported resistance alleles (rym4, rym5, and rym4 $4_{\text {HOR4224 }}$ ) (Perovic et al. 2014; Stein et al. 2005), and 15 new haplotypes for which bymovirus resistance had not been reported before (hap-IX, X, XIII, XIV, XV, XVII, XIX, XXIII, XXIII, XXIV, XXVII, XXVIII, XXXI, XXXVII, XLI, and XLV) (Fig. 3). Wild barley accessions carrying HvEIF4E hap-XLV were also resistant to BaMMV-ASL (Supplementary Table S11), confirming that $H$. spontaneum is a useful source of resistance to bymoviruses. In domesticated barley, sixty-eight (5.31\%) of 1090 accessions carried any of the remaining 18 resistance-conferring HvEIF4E haplotypes (Fig. 3). These haplotypes were found in many barley cultivation areas except the Americas, Near East and Oceania (Fig. 4b), where no reports about the occurrence of the bymovirus disease are available. Interestingly, all resistance-conferring haplotypes contained non-synonymous mutations in the coding sequence of $H v E I F 4 E$. 
Fig. 3 Geographic distribution of barley accessions carrying resistance haplotypes of $H v P$ DIL5- 1 and HvEIF4E against BaMMV-ASL. Bars indicate the total number of accessions carrying the same resistanceconferring haplotype

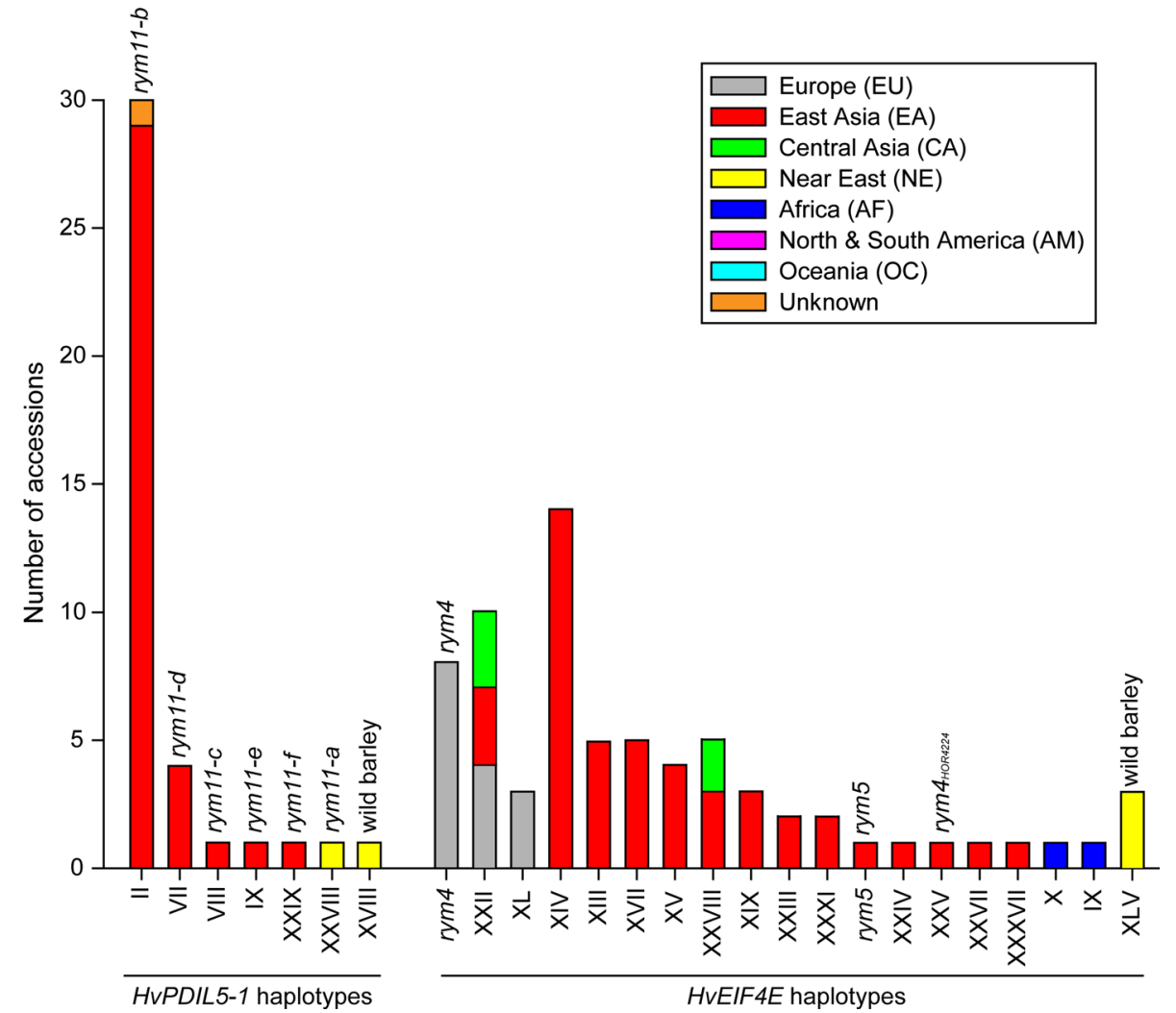

Collectively, the data presented above revealed a strong correlation between bymovirus resistance and either the loss-of-function mutations in HvPDIL5-1 or the non-synonymous mutations in $H v E I F 4 E$. Importantly, resistanceconferring haplotypes were found preferentially in cultivated barley rather than wild barley, suggesting the more frequent rise of bymovirus resistance after domestication.

Type and frequency of mutations in the two host factor genes in context of geographical origins of the domesticated barley accessions.

The large collection of geographically referenced domesticated barley accessions allowed us to investigate if any geographic region contributed disproportionally to gene sequence diversity (loss-of-function mutations of HvPDIL5-1 and non-synonymous mutations of HvEIF4E), and if there were any preferred geographic origins of resistance-conferring haplotypes. Seven sub-populations (Africa, Americas, Central Asia, East Asia, Europe, Near East and Oceania) of the domesticated barley accessions were defined (Supplementary Table S1), and analyzed for diversity in the host factor genes HvPDIL5-1 and HvEIF4E (Tables 2, 3).

\section{HvPDIL5-1}

The polymorphic loci, the number of haplotypes, $H$ and $\pi$ of $H v P D I L 5-1$ decreased in all seven sub-populations of domesticated barley compared to those in wild barley (Tables 1, 2). Six HvPDIL5-1 haplotypes (hap-II, VII, VIII, IX, XXVIII and XXIX) were found to be associated with bymovirus resistance, with five of those containing a lossof-function mutation/deletion and one other carrying a nonsynonymous mutation. One of these resistance-conferring haplotypes of HvPDIL5-1, rym11-a (hap-XXVIII), carrying a 1375-bp deletion was found only in one accession from Near East (Yang et al. 2014b). The other five haplotypes were found in 36 accessions originated from East Asia and one accession (Russia 57, hap-II, rym11-b) for which the exact location of a collection site was uncertain (Fig. 3). Of these, 35 contained haplotypes with loss-offunction mutations, indicating a disproportional frequency of resistance-conferring haplotypes in East Asia compared to other barley cultivation regions (Fig. 4a). The frequency of these haplotypes varied between $0.23 \%$ (e.g. hap-VIII) and $6.56 \%$ (hap-II) in the set of 442 accessions from East Asia used in this study. In comparison, no accessions carrying resistance alleles were found in Europe, where the bymovirus disease is also known to occur (Kühne 2009).

\section{$H v E I F 4 E$}

The genetic diversity of $H v E I F 4 E$ varied among cultivated barley from the different regions of origin (Table 3). The highest diversity was observed in barley from East Asia. 
Fig. 4 Collection sites of the barley accessions carrying resistance-conferring haplotypes of HvPDIL5-1 (a) and $H v E I F 4 E$ (b). Each accession is allocated according to latitude and longitude coordinates of its geographic collection site (represented as red or blue circles). Red and blue circles indicate collection sites for wild and domesticated barleys, respectively. Size of each circle represents the number of accessions collected at each particular collection site (color figure online)

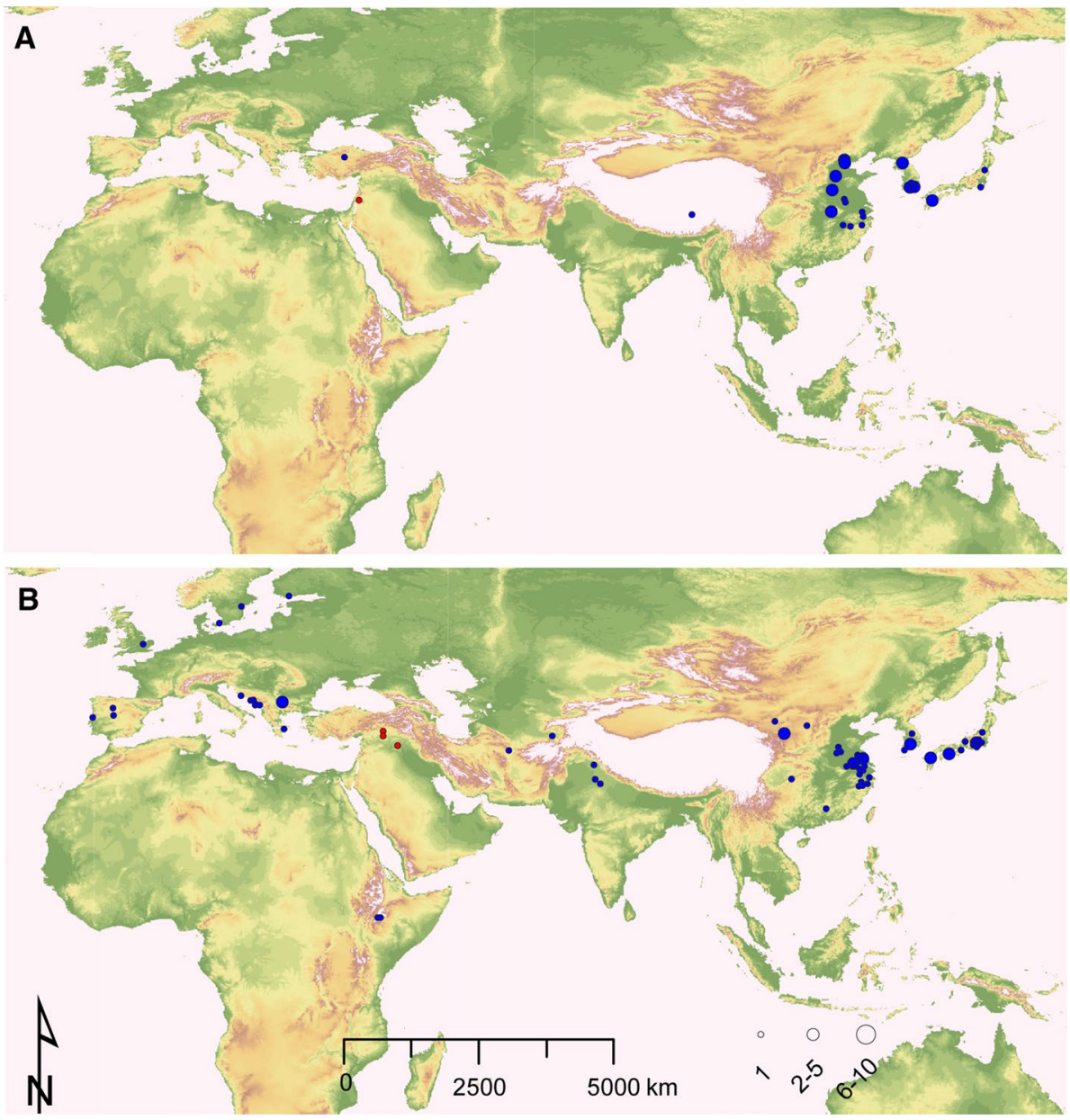

Most of the bymovirus resistance-conferring haplotypes were found exclusively in a single barley cultivation area, except hap-XXII and XXVIII, which were present in more than two major geographic regions (Fig. 3). Two haplotypes, rym 4 and rym5, were extensively found in Europe and East Asia, respectively (Fig. 3). This is consistent with the known origin of barley landraces Ragusa (from Croatia) and Mokusekko 3 (from China) that were originally identified as carriers of rym 4 and rym5, respectively, and used for introgression of bymovirus resistance into elite barley germplasm by breeding (Ordon and Friedt 1993). In East Asia, fourteen resistance-conferring haplotypes were found (Fig. 3). They were present in $46(24.2 \%)$ of 190 cultivated barley accessions from this region in our collection (Table 3). Each haplotype was present at relatively low frequency in the set of accessions tested in this study, ranging between $0.53 \%$ ( 1 of 190, e.g. hap-rym5) and $8.95 \%$ (14 of 190, hap-XIV). By contrast, in Europe only three haplotypes (hap-rym4, XL and XXII) conferring resistance to bymoviruses were found. These haplotypes were present in 15 out of $304(4.93 \%)$ barley accessions from this region (Table 3).
Taken together, the bymovirus resistance-conferring haplotypes caused by loss-of-function mutations in $H v P$ DIL5- 1 and non-synonymous mutations in HvEIF4E were greatly overrepresented in accessions from East Asia (Tables 2, 3), and the predominant occurrence as minor haplotypes together with statistics indicating selection suggest an evolution of bymovirus resistance alleles in barley germplasm from this geographic region.

\section{Discussion}

This work, by combining previously published data and newly generated sequence information, revealed sequence variation in a broad collection of wild and domesticated barley accessions of the two host factor genes, HvEIF4E and HvPDIL5-1, involved in bymovirus infection. Importantly, by testing haplotypes for resistance, this work demonstrated the two distinct types of sequence diversity patterns occurring in resistance forms of the two host factor genes: non-synonymous mutations in HvEIF4E and lossof-function mutations in HvPDIL5-1. Re-sequencing of 


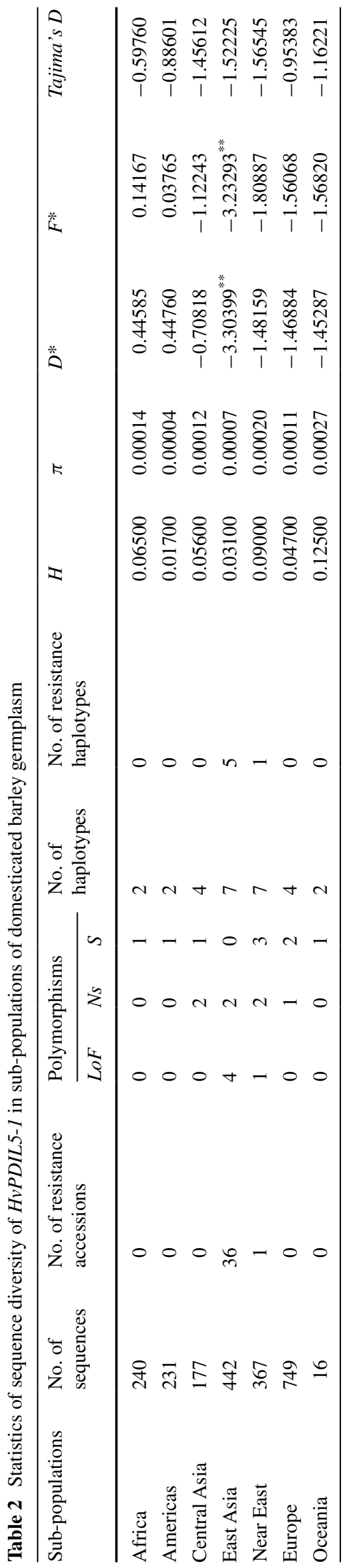

the neighbor genes $H v G T 43$ and HvMCT-1 in a subset of barley accessions indicated that the two distinct patterns of preferential resistance-conferring sequence polymorphism of the two host factor genes were not caused by population demographic history or their chromosomal positions on the respective chromosomes. Haplotypes associated with bymovirus resistance occurred predominantly in domesticated barley accessions but were rare in wild barley, indicating a rapid rise of resistance in cultivated barley post domestication. Interestingly, sources of bymovirus resistance were mainly found in barley germplasm from East Asia where the barley yellow mosaic virus disease is omnipresent (Kühne 2009), indicating the existence of interaction between bymoviruses and host factors. In addition, although the overall resistance spectrum of the identified HvEIF4E and HvPDIL5-1 haplotypes conferring resistance to BaMMV-ASL warrants further investigation (e.g. by testing using a range of BaMMV and BaYMV isolates), these haplotypes represent useful new sources of bymovirus resistance for exploitation in barley breeding programs globally.

The differences in gene diversity patterns observed for HvEIF4E vs HvPDIL5-1 may be explained by the different functions of these two host factor genes.

The eukaryotic translation initiation factor 4E (EIF4E) was repeatedly reported as a host factor involved mainly in potyvirus infections in different plant species (Charron et al. 2008; Wang and Krishnaswamy 2012). The EIF4E protein is of crucial importance in organisms, as it is a component of the conserved eukaryotic translation initiation complex that interacts with the cap-structure of the host mRNAs and enables initiation of the translation of cellular proteins (Browning 1996; Kawaguchi and Bailey-Serres 2002). In previous EcoTILLING (a method for the discovery of natural nucleotide diversity) studies, no haplotypes carrying loss-of-function mutations in EIF4E were found in broad collections of accessions of a number of different crops and related wild plant species (Charron et al. 2008; Hofinger et al.2009, 2011; Ibiza et al. 2010; Konecna et al. 2014; Nieto et al. 2007; Rubio et al. 2009; Stein et al. 2005; Stracke et al. 2007). In the coding sequence of HvEIF4E, only non-synonymous mutations were previously identified in an Ethyl Methane Sulfonate (EMS) induced barley population (Gottwald et al. 2009). Consistent with this, a previous EcoTILLING study using cultivated barley also revealed only non-synonymous and small in-frame insertion/deletion mutations in coding sequence of the HvEIF4E gene (Hofinger et al. 2011). Similarly, in this study surveying the variation in $H v E I F 4 E$ also in a wild barley collection detected only synonymous and non-synonymous mutations and no loss-of-function mutations. This cumulative evidence strongly suggested that $H v E I F 4 E$ is indispensable in barley, although a highly conserved paralogous gene, 
$H v E I F$ (iso) $4 E$, exists in barley and may potentially provide some functional compensation upon loss-of-function of HvEIF4E (Yang et al. 2013). Since the VPg protein of plant viruses in the Potyviridae family plays a role similar to that of the eukaryotic mRNA cap-structure and competes with the plant cellular mRNAs for EIF4E binding (Leonard et al. 2000), the changed compatibility by amino acid changes may block the physical interaction with VPg resulting in disease resistance (Charron et al. 2008). This mechanism reported for potyviruses in an interaction with Arabidopsis may explain the situation in barley, since the amino acid substitutions in either HvEIF4E or the bymovirus VPg protein may influence the efficiency of interaction between these proteins (Habekuß et al. 2008; Kanyuka et al. 2005; Kühne et al. 2003; Stein et al. 2005). Thus, we speculate that amino acid substitutions in HvEIF4E represent the optimal way of evolution of this gene, to maintain the function for protein translation initiation in barley while simultaneously conferring resistance to bymoviruses.

In contrast to the complex evolutionary haplotype network of HvEIF4E in domesticated barley, the HvPDIL5-1 haplotype variation was rather simple. Only 15 haplotypes were found in 2238 cultivated barley accessions. Five haplotypes encoded either no transcript (hap-XXVIII, 1375-bp deletion including first three protein coding exons leads to complete lack of transcript (Yang et al. 2014b), truncated proteins (hap-VII, VIII and XXIX) or shifted coding frames (hap-II). Thus all five identified bymovirus resistance-conferring haplotypes of HvPDIL5-1 lead to non-functional proteins or no protein at all. HvPDIL5-1 belongs to a protein disulfide isomerase (PDI) gene family (Yang et al. 2014b). The number of the family members varied in eukaryotic species. Arabidopsis, rice and maize contain 22, 19 and 22 PDIs, respectively (Houston et al. 2005). Thirty-five barley cultivars from East Asia contained one of these loss-of-function versions of HvPDIL5-1, suggesting that the non-functional alleles unlikely resulted in yield penalties. In addition, the EMS-induced non-functional alleles (rym11-9699 and -10253) of HvPDIL5-1 were not associated with any visible phenotypic changes in plant growth or development (Yang et al. 2014b), suggesting a certain level of dispensability for HvPDIL5-1 in barley. Although an amino acid substitution (ryml1-e) of HvPDIL5-1 can confer bymovirus resistance in domesticated barley, the resistance spectrum of this allele to multiple isolates of bymoviruses still remains to be determined (Yang et al. 2014a). Overcoming resistance conferred by this allele by new bymovirus isolates may be rather likely in contrast to the other loss-of-function alleles (ryml1-a, $b, c, d$, and $f$ ), but this is similar to the situation observed for several HvEIF4E alleles (Kühne et al. 2003; Stein et al. 2005). Complete knock-out of the HvPDIL5-1 gene likely terminates the co-evolution between bymoviruses and this host factor gene. 
Therefore, we postulate that the specific biological functions of both host factors HvPDIL5-1 and HvEIF4E has driven the two observed distinct patterns of gene diversity. Importantly, the highest diversity of the resistance alleles for both host factor genes was found in East Asia, a region with high incidence of barley yellow mosaic virus disease (Kühne 2009).

\section{Did natural selection for bymovirus resistance also occur in European cultivated barley?}

The barley yellow mosaic virus disease occurs in a majority of European countries (Kühne 2009). Given the predominance of resistance-conferring alleles in East-Asian accessions, it is unclear whether natural selection for bymovirus resistances also independently happened in Europe, perhaps even before the active breeding for bymovirus resistance in barley had been initiated (since 1980s). No HvPDIL5-1 haplotypes conferring bymovirus resistance $(r y m 1 / 11)$ were found in the current collection of European barley accessions, supporting the hypothesis that ryml/11 evolved in East Asia. Three HvEIF4E haplotypes (hap-rym4, XXII, and XL) that also confer bymovirus resistance were found in 15 of 304 European barley accessions. The eleven historical accessions (landraces) containing hap-rym 4 or hap-XL were particularly collected before $1970 \mathrm{~s}$ from the Balkans (not shown), a region where the bymovirus disease was not reported until the early 1990s (Katis et al. 1997). The remaining four accessions carrying hap-XXII were from countries of Western and Northern Europe. The same haplotype (hap-XXII) was found also in Central and East Asia. In some geographic regions, where the barley yellow mosaic virus disease was not reported, twelve accessions (including wild and domesticated) showing bymovirus resistance were found. While there was an enrichment of HvEIF4E haplotypes conferring bymovirus resistance in European barley germplasm, from the results of the present study we cannot confidently conclude whether the resistance-conferring mutations in hap-XXII, and hap-XL originated in Europe or were introduced to Europe from elsewhere with the seed trade.

Author contribution statement P.Y., K.K., A.G., F.O. and N.S. designed this research; P.Y., A.H., B.H. and B.K. performed experiments; P.Y. analyzed data; P.Y. and N.S. wrote this manuscript.

Acknowledgements The authors gratefully acknowledge IPK-Genebank (Gatersleben, Germany) and National Small Grains Collection, USDA-ARS (Aberdeen, Idaho, USA) for barley accessions; S. König, I. Walde, J. Perovic, M. Ziems (IPK) and D. Grau (JKI) for excellent technical support; Dr. F. Rabenstein (JKI) for providing BaMMV antibody; Dr. H. Knüpffer (IPK) for helpful discussion; and Dr. Alexander
Walther (University of Gothenburg, Sweden) for Geographic information system (GIS)-based topographical map production. This study was financed by a grant of the German Ministry of Education and Research (BMBF) to N.S. and F.O. ("Plant KBBE II-ViReCrop" FKZ 0315708). B. G. H. was supported by the grant BBE0071981 from the Biotechnology and Biological Sciences Research Council (BBSRC) of the United Kingdom ('Innovation in Crop Science-Exploitation of Genetics for Sustainability' initiative), and K. K. was supported by the BBSRC through the Institute Strategic Program Grant 20:20 Wheat ${ }^{\circledR}$ $(\mathrm{BB} / \mathrm{J} / 00426 \mathrm{X} / 1)$.

Conflict of interest The authors declare that they have no conflict of interest.

Ethical standards The research presented in this work has been generated by following the rules of good scientific practice. The manuscript has not been submitted to more than one journal for simultaneous consideration. The manuscript has not been submitted to more than one journal for simultaneous consideration. No data have been fabricated or manipulated (including images) to support our conclusions. We have acknowledged and cited the work of others to the best of our knowledge. Consent to submit has been received explicitly from all co-authors, as well as from the responsible authorities-tacitly or explicitly-at the institute/organization where the work has been carried out, before the work is submitted.

Open Access This article is distributed under the terms of the Creative Commons Attribution 4.0 International License (http://creativecommons.org/licenses/by/4.0/), which permits unrestricted use, distribution, and reproduction in any medium, provided you give appropriate credit to the original author(s) and the source, provide a link to the Creative Commons license, and indicate if changes were made.

\section{References}

Adams MJ, Swaby AG, Jones P (1988) Confirmation of the transmission of barley yellow mosaic-virus (BaYMV) by the fungus Polymyxa graminis. Ann Appl Biol 112:133-141

Ahlquist P, Noueiry AO, Lee WM, Kushner DB, Dye BT (2003) Host factors in positive-strand RNA virus genome replication. J Virol 77:8181-8186

Browning KS (1996) The plant translational apparatus. Plant Mol Biol 32:107-144

Charron C, Nicolai M, Gallois JL, Robaglia C, Moury BT, Palloix A, Caranta C (2008) Natural variation and functional analyses provide evidence for co-evolution between plant eIF4E and potyviral VPg. Plant J 54:56-68

Chen JP (2005) Progress and prospects of studies on Polymyxa graminis and its transmitted cereal viruses in China. Prog Nat Sci 15:481-490

Clark MF, Adams AN (1977) Characteristics of the microplate method of enzyme-linked immunosorbent assay for the detection of plant viruses. J Gen Virol 34:475-483

Domingo E, Holland JJ (1997) RNA virus mutations and fitness for survival. Annl Rev Microbiol 51:151-178

Gottwald S, Bauer P, Komatsuda T, Lundqvist U, Stein N (2009) TILLING in the two-rowed barley cultivar 'Barke' reveals preferred sites of functional diversity in the gene HvHoxl. BMC Res Notes 2:258

Habekuß A, Kuhne T, Kramer I, Rabenstein F, Ehrig F, Ruge-Wehling B, Huth W, Ordon F (2008) Identification of Barley mild mosaic 
virus isolates in Germany breaking rym5 resistance. J Phytopathol 156:36-41

Hofinger BJ, Jing HC, Hammond-Kosack KE, Kanyuka K (2009) High-resolution melting analysis of cDNA-derived PCR amplicons for rapid and cost-effective identification of novel alleles in barley. Theor Appl Genet 119:851-865

Hofinger BJ, Russell JR, Bass CG, Baldwin T, Dos Reis M, Hedley PE, Li YD, Macaulay M, Waugh R, Hammond-Kosack KE, Kanyuka K (2011) An exceptionally high nucleotide and haplotype diversity and a signature of positive selection for the eIF4E resistance gene in barley are revealed by allele mining and phylogenetic analyses of natural populations. Mol Ecol 20:3653-3668

Houston NL, Fan C, Xiang JQ, Schulze JM, Jung R, Boston RS (2005) Phylogenetic analyses identify 10 classes of the protein disulfide isomerase family in plants, including single-domain protein disulfide isomerase-related proteins. Plant Physiol 137:762-778

Huth W (1991) Verbreitung der Gelbmosaikviren BaYMV, BaMMV und BaYMV-2 und Screening von Gerstensorten auf Resistenz gegenüber BaYMV-2. Nachrichtenbl Dt Pflnzenschutzd 43:5

Ibiza VP, Canizares J, Nuez F (2010) EcoTILLING in capsicum species: searching for new virus resistances. BMC Genom 11:631

Ikata S, Kawai I (1940) Studies on wheat yellow mosaic disease. Noji Kairyo Shiryo 154:1-123

International barley research consortium (2012) A physical, genetic and functional sequence assembly of the barley genome. Nature 491:711-716

Jones JDG, Dangl JL (2006) The plant immune system. Nature 444:323-329

Kai H, Takata K, Tsukazaki M, Furusho M, Baba T (2012) Molecular mapping of Rym17, a dominant and rym18 a recessive barley yellow mosaic virus (BaYMV) resistance genes derived from Hordeum vulgare L. Theor Appl Genet 124:577-583

Kang BC, Yeam I, Jahn MM (2005) Genetics of plant virus resistance. Annu Rev Phytopathol 43:581-621

Kanyuka K, Ward E, Adams MJ (2003) Polymyxa graminis and the cereal viruses it transmits: a research challenge. Mol Plant Pathol 4:393-406

Kanyuka K, Druka A, Caldwell DG, Tymon A, McCallum N, Waugh R, Adams MJ (2005) Evidence that the recessive bymovirus resistance locus rym4 in barley corresponds to the eukaryotic translation initiation factor 4E gene. Mol Plant Pathol 6:449-458

Katis N, TzavellaKlonari K, Adams MJ (1997) Occurrence of barley yellow mosaic and barley mild mosaic bymoviruses in Greece. Eur J Plant Pathol 103:281-284

Kawaguchi R, Bailey-Serres J (2002) Regulation of translational initiation in plants. Curr Opin Plant Biol 5:460-465

Konecna E, Safarova D, Navratil M, Hanacek P, Coyne C, Flavell A, Vishnyakova M, Ambrose M, Redden R, Smykal P (2014) Geographical gradient of the $e I F 4 E$ alleles conferring resistance to potyviruses in Pea (Pisum) Germplasm. PLoS ONE 9:e90394

Konishi T, Ban T, Iida Y, Yoshimi R (1997) Genetic analysis of disease resistance to all strains of BaYMV in a Chinese barley landrace, Mokusekko 3. Theor Appl Genet 94:871-877

Kühne T (2009) Soil-borne viruses affecting cereals-known for long but still a threat. Virus Res 141:174-183

Kühne T, Shi N, Proeseler G, Adams MJ, Kanyuka K (2003) The ability of a bymovirus to overcome the rym4-mediated resistance in barley correlates with a codon change in the VPg coding region on RNA1. J Gen Virol 84:2853-2859

Lee K-J, Kashiwazaki S, Hibi T, So I-Y (1996) Properties and capsid protein gene sequence of a Korean isolate of barley. Ann Phytopathol Soc Jpn 62:397-401

Leonard S, Plante D, Wittmann S, Daigneault N, Fortin MG, Laliberte JF (2000) Complex formation between potyvirus VPg and translation eukaryotic initiation factor $4 \mathrm{E}$ correlates with virus infectivity. J Virol 74:7730-7737

Librado P, Rozas J (2009) DnaSP v5: a software for comprehensive analysis of DNA polymorphism data. Bioinformatics 25:1451-1452

Nieto C, Piron F, Dalmais M, Marco CF, Moriones E, Gomez-Guillamon ML, Truniger V, Gomez P, Garcia-Mas J, Aranda MA, Bendahmane A (2007) EcoTILLING for the identification of allelic variants of melon eIF4E, a factor that controls virus susceptibility. BMC Plant Biol 7:34

Obbard DJ, Dudas G (2014) The genetics of host-virus coevolution in invertebrates. Curr Opin Virol 8C:73-78

Oerke EC (2006) Crop losses to pests. J Agr Sci 144:31-43

Okada Y, Kashiwazaki S, Kanatani R, Arai S, Ito K (2003) Effects of barley yellow mosaic disease resistant gene ryml on the infection by strains of barley yellow mosaic virus and Barley mild mosaic virus. Theor Appl Genet 106:181-189

Ordon F, Friedt W (1993) Mode of inheritance and genetic diversity of bammv resistance of exotic barley germplasms carrying genes different from $Y m 4$. Theor Appl Genet 86:229-233

Ordon F, Ahlemeyer J, Werner K, Kohler W, Friedt W (2005) Molecular assessment of genetic diversity in winter barley and its use in breeding. Euphytica 146:21-28

Perovic D, Kramer I, Habekuß A, Perner K, Pickering R, Proeseler G, Kanyuka K, Ordon F (2014) Genetic analyses of BaMMV/ BaYMV resistance in barley accession HOR4224 result in the identification of an allele of the translation initiation factor $4 \mathrm{e}$ (Hv-eIF4E) exclusively effective against Barley mild mosaic virus (BaMMV). Theor Appl Genet 127:1061-1071

Plumb RT, Lennon EA, Gutteridge RA (1986) The effects of infection by barley yellow mosaic-virus on the yield and components of yield of barley. Plant Pathol 35:314-318

Rausher MD (2001) Co-evolution and plant resistance to natural enemies. Nature 411:857-864

Robaglia C, Caranta C (2006) Translation initiation factors: a weak link in plant RNA virus infection. Trends Plant Sci 11:40-45

Ruan YL, Jin DD, Xu RG, Gao DS, Feng ZJ, Ma YM (1984) The identification of resistance in barley varities to the barley yellow mosaic virus (BaYMV). Acta Phytophy Sin 11:217-222

Rubio M, Nicolai M, Caranta C, Palloix A (2009) Allele mining in the pepper gene pool provided new complementation effects between pvr2-eIF4E and pvr6-eIF(iso) $4 E$ alleles for resistance to pepper veinal mottle virus. J Gen Virol 90:2808-2814

Sacristan S, Garcia-Arenal F (2008) The evolution of virulence and pathogenicity in plant pathogen populations. Mol Plant Pathol 9:369-384

Soosaar JLM, Burch-Smith TM, Dinesh-Kumar SP (2005) Mechanisms of plant resistance to viruses. Nat Rev Microbiol 3:789-798

Stein N, Perovic D, Kumlehn J, Pellio B, Stracke S, Streng S, Ordon F, Graner A (2005) The eukaryotic translation initiation factor $4 \mathrm{E}$ confers multiallelic recessive bymovirus resistance in Hordeum vulgare (L.). Plant J 42:912-922

Stracke S, Presterl T, Stein N, Perovic D, Ordon F, Graner A (2007) Effects of introgression and recombination on haplotype structure and linkage disequilibrium surrounding a locus encoding bymovirus resistance in barley. Genetics 175:805-817

Stukenbrock EH, McDonald BA (2008) The origins of plant pathogens in agro-ecosystems. Annu Rev Phytopathol 46:75-100

Tanksley SD, McCouch SR (1997) Seed banks and molecular maps: Unlocking genetic potential from the wild. Science 277:1063-1066

Tiffin P, Moeller DA (2006) Molecular evolution of plant immune system genes. Trends Genet 22:662-670 
Wang AM, Krishnaswamy S (2012) Eukaryotic translation initiation factor 4E-mediated recessive resistance to plant viruses and its utility in crop improvement. Mol Plant Pathol 13:795-803

Whitham SA, Wang Y (2004) Roles for host factors in plant viral pathogenicity. Curr Opin Plant Biol 7:365-371

Yang P, Perovic D, Habekuß A, Zhou RN, Graner A, Ordon F, Stein N (2013) Gene-based high-density mapping of the gene rym7 conferring resistance to Barley mild mosaic virus (BaMMV). Mol Breed 32:27-37

Yang P, Habekuß A, Ordon F, Stein N (2014a) Analysis of bymovirus resistance genes on proximal barley chromosome $4 \mathrm{HL}$ provides the basis for precision breeding for BaMMV/BaYMV resistance. Theor Appl Genet 127:1625-1634

Yang P, Lupken T, Habekuß A, Hensel G, Steuernagel B, Kilian B, Ariyadasa R, Himmelbach A, Kumlehn J, Scholz U, Ordon F, Stein N (2014b) PROTEIN DISULFIDE ISOMERASE LIKE 5-1 is a susceptibility factor to plant viruses. Proc Natl Acad Sci USA 111:2104-2109

Zhou XK, Cao MS (1985) Screening tests of barley cultivars for resistance to barley yellow mosaic virus. Acta Phytophy Sin $12: 217-223$ 